\title{
Maternal imprinting, mitochondrial DNA, nuclear DNA and Alzheimer's disease
}

\author{
Alberto Pérez-Mediavilla ${ }^{1,2,3}$, Marta Zamarbide ${ }^{1,2}{ }^{*}$ (1) \\ ${ }^{1}$ Neuroscience Program, Center for Applied Medical Research (CIMA), University of Navarra, 31008 Pamplona, Spain \\ ${ }^{2}$ Instituto de Investigación Sanitaria de Navarra (IDISNA), 31008 Pamplona, Spain \\ ${ }^{3}$ Department of Biochemistry and Genetics, University of Navarra, 31008 Pamplona, Spain
}

*Correspondence: Marta Zamarbide, Neuroscience Program, Center for Applied Medical Research (CIMA), University of Navarra, 31008 Pamplona, Spain. mzamarbide@unav.es

Academic Editor: Shile Huang, Louisiana State University Health Science Center, USA

Received: August 20, 2021 Accepted: October 14, 2021 Published: October 29, 2021

Cite this article: Pérez-Mediavilla A, Zamarbide M. Maternal imprinting, mitochondrial DNA, nuclear DNA and Alzheimer's disease. Explor Neuroprot Ther. 2021;1:121-6. https://doi.org/10.37349/ent.2021.00010

\begin{abstract}
Familial early-onset Alzheimer's disease (AD) is more probable in individuals coming from mothers diagnosed with AD than from fathers diagnosed with AD. Studies in animal models have shown maternal imprinting due to the transmission to the embryo of altered material in the ovum. In the case of transgenic animals harboring a mutated form of the human amyloid precursor protein (APP), offspring from crosses with wild-type (WT) fathers and transgenic mothers display more abnormalities than offspring from crosses with transgenic fathers and WT mothers. Expression of the mutated APP in the ovum may lead to alterations that may be genetic and/or epigenetic in the nuclear and/or the mitochondrial DNA. These modifications that are transmitted to the new living beings affect more mitochondrial proteins and, therefore, the mitochondrial function may be affected in adulthood by trends present in the ovum.
\end{abstract}

\section{Keywords}

$\beta$-amyloid, protein aggregates, synuclein, fibrillary tangles, mitochondria, ovum, mitophagy

\section{Impact of mother's ovum material on the risk of Alzheimer's disease in offspring}

The embryo results from a female ovum that is fertilized by a spermatozoon from a male. Whereas a fertilizing spermatozoon only provides the nucleus, the ovum provides all its components to the embryo. Hereditary diseases mainly come from mutations in the nuclear genome but they may come from mutations in the mitochondrial DNA (mtDNA) of the ovum. Hereditary mitochondrial diseases may be transmitted by either parent and, actually, the diagnosis may not be an easy task [1]. Surely, mitochondrial diseases may not be related to mtDNA as there is nuclear DNA that codes for proteins needed by mitochondria. However, there are well-characterized diseases with maternal transmission and the mutation responsible for the disease occurs in mtDNA.

As atypical as it may seem, it is relevant to consider whether the general state of the ovum that is being fertilized may influence the risk of the new living being suffering from any disease. The state of the 
spermatozoa is virtually irrelevant as only paternal nuclei are transmitted to the progeny. In summary, can ovarian cells that are altered by any reason influence the risk of offspring suffering from, among others, neurodegenerative diseases?

A study in mice shows that maternal material of mothers who carry a transgene for a familial mutation in the human amyloid precursor protein (APP) increases the risk of suffering from cognitive impairments despite that offspring does not carry the transgene, i.e. in animals that are controls of the transgenic $\operatorname{Tg} 2576$ line, which is a well characterized mice model of Alzheimer's disease (AD). Specifically, the Tg2576 mice that carry in heterozygosity the human APP (695 amino-acid-long isoform) with the Swedish double mutation (Met671 $\rightarrow$ Leu and Lys670 $\rightarrow$ Asn) $\left(\mathrm{APP}_{\text {Swe }}\right.$ ), display cognitive deficits starting at around 8-9 months of age [2]. A study was undertaken to compare heterozygous animals from mothers carrying or not the transgene led to very interesting results [3]. On the one hand, the $\mathrm{AD}$-associated alteration to $\mathrm{AD}$ pathology was aggravated in offspring coming from mothers carrying the transgene. On the other hand, AD-like pathology was found in the offspring of mothers carrying the transgene but that were not themselves carrying the transgene. These results show that alterations due to mutated APP are maternally transmitted to mice that should be healthy as they do not carry any APP-related mutation. In summary, something in the cell of the ovum is transmitted in such a way that affects the phenotype of offspring. This finding is consistent with a maternal inheritance trend, namely, familial $\mathrm{AD}$, which is more probable in individuals coming from mothers diagnosed with $\mathrm{AD}$ than from fathers diagnosed with $\mathrm{AD}$ [4-7]. Therefore, the purpose of this review is to highlight the relevance of maternal imprinting and specifically the influence of the mitochondria in the possibility of developing early-onset familial AD.

\section{Underlying mechanism of maternal transmission of traits}

One possibility is that mutated APP acts like a prion and confers an abnormal structure that leads to protein aggregation. Although prions are one of the potential causes of AD, such possibility is unlikely for this type of maternal transmission. If it were the case, embryos could be non-viable and/or neonates would already have cognitive deficits, i.e. it is expected that a prion-like scenario would lead to symptoms at the embryo level and upon birth. Therefore, other possibilities must be contemplated, being the most obvious alteration in the genetic material of the ovum.

Surely, mutated APP may lead to alterations in the DNA, either at the nuclear or the mitochondrial level. These mutations would be transmitted to the progeny and become silent as exome determination is not any procedure of routine in $\mathrm{AD}$. However, in familial cases of $\mathrm{AD}$, there is not any evidence of mutations in "random" genes, i.e. mutated-APP-induced mutations should have been detected at some point at least for some of the early-onset familial AD cases. At this point, it is tempting to speculate that any mutation in APP of familial cases transmitted by the mother may lead to epigenetic alterations in the DNA of the ovum. These epigenetic traits could condition the overall performance of the progeny and would be difficult to detect. This possibility is attractive as several studies report epigenetic alterations in AD patients and animal AD models.

\section{Mitochondria in neurodegenerative diseases}

Mitochondria are at the center of the metabolism in mammalian cells. As a main source of energy in mammals, carbohydrates may undergo anaerobic glycolysis but it is also possible, via mitochondrial Krebs' cycle and electron transport chain, to be fully oxidized to $\mathrm{CO}_{2}$ and water. Actually, neurons are completely dependent on the full oxidation of glucose to be healthy. Only in extreme conditions, neurons may also rely on the energy provided by ketone bodies. Even in the case of needing the use of ketone bodies, their oxidation requires the action of neuronal mitochondria. Finally, mitochondria are at the center stage in terms of coping and/or producing oxidative stress. Expenditure of glucose and oxygen by the brain is much higher than that of many other tissues in the human body.

There is cumulative evidence of mitochondrial alteration in many neurodegenerative diseases. The cause of the disruption and the details of how the mitochondria are disrupted can vary from disease to disease. Although the resistance of the mitochondria is remarkable, any reduction in efficiency, in the long term, leads 
to serious problems that can result in cell death. This possibility is especially problematic in the case of neurons that are highly differentiated cells which cannot be replaced; furthermore, the degree of neurogenesis in the adult is very limited. In summary, it appears that for many neurodegenerative diseases, faulty mitochondria may be a consequence of the disease, but it is often the cause of neuronal death.

Cause or consequence, alterations in mitochondrial structure, biogenesis and/or function have been detected in immunohistochemical and/or molecular studies performed in patient samples or AD models. A few examples encompass oxidative stress affecting mitochondrial function to mitochondria-endoplasmic reticulum tethering defects, protein-import deficits and mitochondrial calcium dyshomeostasis [8-11]. A more complete review may be found elsewhere [12]. It should be noted that there are also examples in late-onset cases with maternal family history such as a reduced mitochondrial cytochrome oxidase [13]. Moreover, consequences of maternal inheritance include mutations in genes in the X chromosome and mtDNA [14].

In addition, it must be taken into account that aging is the primary risk factor for the development of a neurodegenerative disease and compromised autophagy is a hallmark of aging. Mitophagy, the main by which damaged mitochondria are degraded, plays a fundamental role in protecting neurons from aging-related events [15]. On keeping control over the clearance of damaged mitochondria, mitophagy ensures a healthy mitochondrial pool in the high-energy demanding axon terminals and enables normal neuronal function and homeostatic neuronal plasticity [16]. Added to the inefficient elimination of damaged mitochondria via mitophagy, a leakage of molecules to the cytosol increases neuronal dysfunction, neuroinflammation and cell death [17]. Moreover, in AD models, mitophagy diminishes the level of insoluble amyloid beta 1-42 (Aß1-42) and $A \beta 1-40$ and prevents cognitive impairment through microglial phagocytosis of extracellular $A \beta$ plaques [18].

\section{APP, ovum and mitochondrial alterations}

Mutations in APP or Tau genes may lead to somatic mutations or epigenetic modifications that if occurring in the ovum could be transmitted to the progeny. For instance, is APP expressed in the ovum? APP is expressed in the porcine ovary [19] and a recent report indicates that, in ovarian cancer cells, APP is the target of miR-20a [20]. Returning to the Tg2576 model, Cuadrado-Tejedor and colleagues show that in adult heterozygous mice compared with control non-transgenic littermates, the highest percentage of proteins whose levels were altered were mitochondrial proteins [21]. Therefore, the above-mentioned results of maternal imprinting of $\mathrm{Tg} 2576$ animals can be due to mutant- $A P P$-induced genetic and/or epigenetic changes in genes coding for mitochondrial proteins.

Lately, there is a tendency to study neurodegenerative diseases from the point of view of what they have in common, i.e. the accumulation/aggregation of proteins: huntingtin in Huntington's disease, alpha-synuclein in Parkinson's disease, and APP and Phospho-Tau (pTau) in AD. A recent study comparing the consequences (in animal models) of mutations related to familial cases of Parkinson's disease has led to two main conclusions that can serve to understand the etiopathological mechanisms operating in sporadic cases of the disease [22]. Aging may lead to failure to appropriately process alpha-synuclein thus leading to protein aggregation and Lewy body formation. Alternatively, mutant forms of the protein may, upon a time, exhaust the machinery to properly address the processing of such mutated proteins thus leading to protein aggregation. Interestingly, the study convincingly indicates that mitochondria are located at the center of the two alternative mechanisms. Something similar could occur for APP or pTau and AD.

\section{Conclusions}

The above-mentioned study, showing maternal imprinting and that ovum material can impact on the risk of $\mathrm{AD}$ and/or aggravate the symptoms, also showed that the mitochondrial voltage-dependent anion channel 1 (VDAC1) was increased in Tg2576 animals if compared with non-transgenic animals. More importantly, the level of VDAC1 in WT offspring from affected mothers was also increased (when compared to actual WT animals). Mitochondrial porin, or VDAC, is "a multi-functional mitochondrial protein regulating cell life and death" [23]. Accordingly, any alteration in the level of this protein in the ovum (as in Tg2576 mothers carrying the $\mathrm{APP}_{\text {Swe }}$ ) is transmitted to the 
mitochondria of embryonic cells and results in affecting the mitochondrial function of the new living being. Maternal imprinting consisting of early-onset and aggravated cognitive deficits in animals that do not harbor the human $A P P$ transgene seems to be due to genetic and/or epigenetic alterations that are transmitted to the embryo and all the way up to the new living being. Mitochondrial endurance makes it possible to cope with metabolic and stress-releasing functions upon birth and up to young adulthood but not upon further aging. If apart from epigenetic alterations inherited at the embryonic stage, the animal carries the human $A P P$ transgene mitochondria surrender earlier than in offspring coming from WT mothers, i.e. symptoms appear earlier and are more aggravated when age-matched animals are compared.

To sum up, any alteration that occurs at the mitochondrial level in the ovum will be transmitted to the embryonic cells and affect the new living being. This fact, added to genetic alterations associated with AD, accelerates and aggravates the disease. On the other hand, these same mitochondrial alterations during youth may not affect the human being given the natural endurance of the organelle. However, any small alteration in maternal mitochondria "inherited" by the progeny may lead to the alteration of different processes such as mitophagy; accordingly, aging may lead to the early appearance of neurodegenerative diseases, not associated with any nuclear genetic factor such as it occurs in sporadic AD.

\section{Abbreviations}

Aß1-42: amyloid beta 1-42

AD: Alzheimer's disease

APP: amyloid precursor protein

mtDNA: mitochondrial DNA

VDAC1: voltage-dependent anion channel 1

WT: wild-type

\section{Declarations}

Author contributions

MZ designed the manuscript. APM and MZ searched for suitable papers and wrote the manuscript.

\section{Conflicts of interest}

The authors declare that they have no conflicts of interest.

\section{Ethical approval}

Not applicable.

\section{Consent to participate}

Not applicable.

\section{Consent to publication}

Not applicable.

\section{Availability of data and materials}

Not applicable.

\section{Funding}

Not applicable.

\section{Copyright}

(c) The Author(s) 2021. 


\section{References}

1. Jackson CB, Turnbull DM, Minczuk M, Gammage PA. Therapeutic manipulation of mtDNA heteroplasmy: a shifting perspective. Trends Mol Med. 2020;26:698-709.

2. Hsiao K, Chapman P, Nilsen S, Eckman C, Harigaya Y, Younkin S, et al. Correlative memory deficits, Abeta elevation, and amyloid plaques in transgenic mice. Science. 1996;274:99-102.

3. Zamarbide M, Gil-Bea FJ, Bannenberg P, Martínez-Pinilla E, Sandoval J, Franco R, et al. Maternal imprinting on cognition markers of wild type and transgenic Alzheimer's disease model mice. Sci Rep. 2018;8:6364.

4. Edland SD, Silverman JM, Peskind ER, Tsuang D, Wijsman E, Morris JC. Increased risk of dementia in mothers of Alzheimer's disease cases: evidence for maternal inheritance. Neurology. 1996;47:254-6.

5. Ehrenkrantz D, Silverman JM, Smith CJ, Birstein S, Marin D, Mohs RC, et al. Genetic epidemiological study of maternal and paternal transmission of Alzheimer's Disease. Am J Med Genet. 1999;88:378-82.

6. Honea RA, Swerdlow RH, Vidoni ED, Goodwin J, Burns JM. Reduced gray matter volume in normal adults with a maternal family history of Alzheimer disease. Neurology. 2010;74:113-20.

7. Mosconi L, Brys M, Switalski R, Mistur R, Glodzik L, Pirraglia E, et al. Maternal family history of Alzheimer's disease predisposes to reduced brain glucose metabolism. Proc Natl Acad Sci U S A. 2007;104:19067-72.

8. Islam MT. Oxidative stress and mitochondrial dysfunction-linked neurodegenerative disorders. Neurol Res. 2017;39:73-82.

9. Lee SH, Lutz D, Mossalam M, Bolshakov VY, Frotscher M, Shen J. Presenilins regulate synaptic plasticity and mitochondrial calcium homeostasis in the hippocampal mossy fiber pathway. Mol Neurodegener. 2017;12:48.

10. Goyal S, Chaturvedi RK. Mitochondrial protein import dysfunction in pathogenesis of neurodegenerative diseases. Mol Neurobiol. 2021;58:1418-37.

11. Liu Y, Zhu X. Endoplasmic reticulum-mitochondria tethering in neurodegenerative diseases. Transl Neurodegener. 2017;6:21.

12. Macdonald R, Barnes K, Hastings C, Mortiboys H. Mitochondrial abnormalities in Parkinson's disease and Alzheimer's disease: can mitochondria be targeted therapeutically? Biochem Soc Trans. 2018;46:891-909.

13. Mosconi L, de Leon M, Murray J, E L, Lu J, Javier E, et al. Reduced mitochondria cytochrome oxidase activity in adult children of mothers with Alzheimer's disease. J Alzheimers Dis. 2011;27:483-90.

14. Mosconi L, Berti V, Swerdlow RH, Pupi A, Duara R, de Leon M. Maternal transmission of Alzheimer's disease: prodromal metabolic phenotype and the search for genes. Hum Genomics. 2010;4:170-93.

15. Aman Y, Schmauck-Medina T, Hansen M, Morimoto RI, Simon AK, Bjedov I, et al. Autophagy in healthy aging and disease. Nature Aging. 2021;1:634-50.

16. Kobro-Flatmoen A, Lagartos-Donate MJ, Aman Y, Edison P, Witter MP, Fang EF. Re-emphasizing early Alzheimer's disease pathology starting in select entorhinal neurons, with a special focus on mitophagy. Ageing Res Rev. 2021;67:101307.

17. Sliter DA, Martinez J, Hao L, Chen X, Sun N, Fischer TD, et al. Parkin and PINK1 mitigate STING-induced inflammation. Nature. 2018;561:258-62.

18. Fang EF, Hou Y, Palikaras K, Adriaanse BA, Kerr JS, Yang B, et al. Mitophagy inhibits amyloid- $\beta$ and tau pathology and reverses cognitive deficits in models of Alzheimer's disease. Nat Neurosci. 2019;22:401-12.

19. Kimura A, Kakinuma K, Yonezawa S, Takabashi T. Expression of $\beta$-amyloid precursor protein in the porcine ovary. Zool Sci. 2000;17:769-77.

20. Fan X, Liu Y, Jiang J, Ma Z, Wu H, Liu T, et al. MiR-20a promotes proliferation and invasion by targeting APP in human ovarian cancer cells. Acta Biochim Biophys Sin (Shanghai). 2010;42:318-24. 
21. Cuadrado-Tejedor M, Cabodevilla JF, Zamarbide M, Gómez-Isla T, Franco R, Perez-Mediavilla A, et al. Age-related mitochondrial alterations without neuronal loss in the hippocampus of a transgenic model of Alzheimer's disease. Curr Alzheimer Res. 2013;10:390-405.

22. Franco R, Rivas-Santisteban R, Navarro G, Pinna A, Reyes-Resina I. Genes implicated in familial Parkinson's disease provide a dual picture of nigral dopaminergic neurodegeneration with mitochondria taking center stage. Int J Mol Sci. 2021;22:4643.

23. Shoshan-Barmatz V, De Pinto V, Zweckstetter M, Raviv Z, Keinan N, Arbel N. VDAC, a multi-functional mitochondrial protein regulating cell life and death. Mol Aspects Med. 2010;31:227-85. 